\title{
BEACON TECHNOLOGY FOR REAL-TIME INFORMING THE TRAFFIC NETWORK USERS ABOUT THE ENVIRONMENT
}

\author{
Marko PERIŠA, Ivan CVITIĆ, Dragan PERAKOVIĆ ${ }^{*}$ Siniša HUSNJAK \\ Faculty of Transport and Traffic Sciences, University of Zagreb, Croatia
}

Received 17 October 2016; revised 22 October 2017; accepted 19 December 2017

\begin{abstract}
Informing the users about their environment is of extreme importance for their full and independent functioning in the traffic system. Today's development of technology provides the user the access to information about their environment by using the smartphone device at any moment if there is a defined applicative solution. For this, it is necessary to define the user's environment according to the Ambient Assisted Living (AAL) concept, which understands adequate technology of gathering, processing and distribution of information. This paper presents the proposal of the solution for informing the traffic network users about the environment for the defined group of users based on the beacon technology. The mentioned solution is based on the results of two separate studies about the needs of users who move along a part of the traffic network. The aim of the proposed solution is to provide the user with precise and real-time information and to raise the level of safety during movement.
\end{abstract}

Keywords: ambient assisted living, wireless technologies, information management system, assistive technology, identification, traffic network.

\section{Introduction}

Current informing of traffic system users can be improved by the application of advanced information and communication systems, i.e. by synergy of the service functionality of mobile communication networks and applications on smartphone devices. One of the examples of informing the user who is in the role of a pedestrian is the smartphone application and Global Positioning System (GPS) technology. The problem of such a form of informing is defining the Points Of Interest (POI) location that provide the user with the ambient information, where they can experience deviations of even up to $40 \mathrm{~m}$, (Periša 2013; Peraković et al. 2015b). The aim of this research is to raise the level of safety for the user moving along a traffic network by using advanced information and communication solutions.

The target group of users in this research are persons with impaired vision (blind and visually impaired) and elderly groups of users in the age of 65+ whose safety of movement can be additionally endangered by the problem of determining the location and provision of information related with it. This group of users represents the risk group due to reduced level of functionality in the decision-making processes while moving along the traffic network.
The research has been directed to the identification of parameters (users' requirements) for the definition of users informing services while moving along the part of the traffic network. A part of the traffic network consists of approaches to bus and railway stations, gathering places (squares) and traffic intersections and public urban transport stops (tram and bus). The application of Ambient Assisted Living (AAL) concept can enable the users who use smartphones to use precise and real-time information on the environment and facilitate safe and reliable navigation by using the user equipment.

The analysis of wireless communications technology has defined the optimal technology with minimal deviations $(<1 \mathrm{~m})$ for providing real-time information to the user of informing system. The research was carried out in the area of daily user movement with all elements surrounding the user (pedestrian zone of movement, tram and bus station and traffic intersection). Apart from the mentioned elements, his environment consists of all POI such as: cultural sights, sports facilities, public facilities and objects of various contents. For accurate informing and routing of the user moving on the part of the traffic network, user routing simulation was performed us-

${ }^{*}$ Corresponding author. E-mail: dragan.perakovic@fpz.hr 
ing beacon transmitters in the MATLAB programming environment. The synergy of modern technologies in the Cloud Computing (CC) environment can offer a service of routing and real-time informing for blind and visually impaired persons and older persons when moving within a part of the traffic network.

\section{Previous research}

Previous studies in this area encompass exclusively the area of AAL concept application in the closed environment and in order to follow the activities of the users in the area of medicine (Garcia, Rodrigues 2015; Brinkmann et al. 2008). The mentioned literature has also dealt with the area of data transfer in AAL concept to the end user. The AAL concept encompasses the application of wireless communication technologies such as Radio-Frequency IDentification (RFID), Near-Field Communication (NFC), Bluetooth, Wi-Fi in order to collect and process data and provide the requested information to the end user. For this purpose, the structure of information recorded in tags is important if this refers to NFC or RFID technology (Peraković et al. 2015a).

For using NFC technology in order to inform the users it is necessary to use the mobile applications on the user smartphone device, which means that it is important to customise the interface (Spinsante, Gambi 2015). For accurate guidance and navigation of users who use mobile devices today there is an array of applicative solutions based on GPS technology. The drawback that is imposed by the mentioned technology is the inaccuracy in reading the POI location, which can be even up to $40 \mathrm{~m}$ in the mentioned research. By using Location-Based Services (LBS) based on several different technologies (NFC, Bluetooth and GPS) the users can get accurate information about their environment (Cheng et al. 2016; Periša et al. 2015). Authors who researched the area of application of GPS technology for guiding and informing users focused their solutions for designing new aids (Brilhault et al. 2011). New aids as such are not designed according to the basic principles of universal design and represent a technically unacceptable solution for the users. GPS-based user guidance systems often have location-specific errors, which further jeopardizes the user's movement safety. Because of the mentioned disadvantages of GPS technology in determining the location of a user, it is possible to use economically more acceptable RFID and ultrasonic technology as one of the aided technologies (Dhananjeyan et al. 2016; Loomis et al. 2016). Researchers suggest an economically viable solution for blind and visually impaired persons, but lack of research are undefined user requirements and no review of the proposed solution with system users. User requirements define the required functionalities of the informing service from which it is possible to define system architecture elements required for its delivery. Technology development and increasing use of new concepts such as the Internet of Things (IoT) and CC implies research of the possibilities of their application for the purpose of informing the user when moving in an external environment (Korial, Abdullah 2016). Object identification technologies in whose class, except Automatic Identification and Data Capture (AIDC), belong beacon transmitters have an increasing presence in numerous researches (Boualouache et al. 2015; Van der Bie et al. 2016). The aforementioned identification technologies are highly suitable for use in the IoT concept due to their technical characteristics and economic feasibility. Visually impaired persons use a white cane as a basic aid tool. Research on the possibility of upgrading the white cane with sensor technology is possible according to the proposed architecture, which enables better users informing in the external environment (Ramirez et al. 2017). Using the users location data available through social networks and sensors in the IoT environment, it is also possible to develop a help system for informing the visually impaired (Joseph et al. 2015).

This research will propose a system architecture based on CC concept to provide accurate and real-time information to the user by using beacon technology. The architecture of the system will be based on the user requirements while moving along a part of the traffic network and it will encompass the guidelines of designing the service for smartphone devices.

\section{Methodology of research}

In this work, two separate studies have been done about the users' needs who move along a part of the traffic network. In order to define the user requirements according to the quality of providing accurate information a study was carried out in nine homes for the elderly and disabled persons and with visually impaired persons.

A study was also carried out on a part of the traffic network along which the users move every day in order to analyse all the relevant information about the user environment necessary for safe movement and informing (Figure 1). Blue points define the locations equipped by devices that allow identification of users and real-time informing about the environment. In order to provide such information, the beacon BLE Bluetooth $v 4.0$ devices were used.

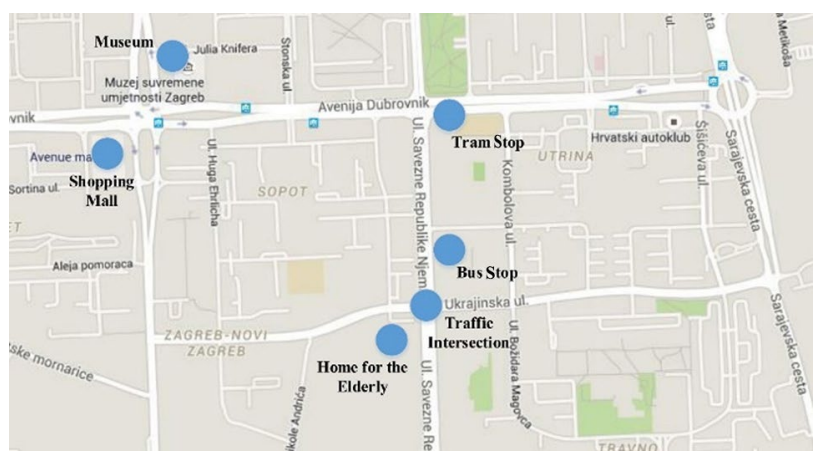

Figure 1. Research locations 


\subsection{Research of the elderly users' needs}

The research was carried out in the homes for the elderly and disabled and included 209 respondents out of which $73 \%$ females and $27 \%$ males in the form of a questionnaire. The questions were directed to the possibility of using new services based on Information and Communications Technology (ICT) technologies. Figure 2 shows the function in which the users would use the new service out of which $47 \%$ of users opted for the orientation and movement that includes user information.

There are $75 \%$ of users using mobile terminal devices out of which $78 \%$ are feature phone, smartphone $14 \%$ and the device adapted to the elderly $8 \%$. The reason for this high percentage of using the feature phone device is the most frequent purpose of usage, and that is calling $99.4 \%$. While carrying out the survey the users were presented the possibilities of using the services for smartphone devices and $55.7 \%$ users stated that they would like to learn the functionalities of smartphone devices. In everyday activities $78 \%$ of users want to use their mobile terminal device.

The user equipment that would be used by the users in their everyday needs is shown in Figure 3, where 108 users expressed their wish to use the bracelet, which has the functionalities of a smartphone device. There are $53.1 \%$ of users who want the users informing service in the systems of urban public transport such as bus or tram. The informing service for the elderly should be designed according to the universal design principles. Elderly persons also have the need for using assistive aids in carrying out everyday activities (Häggblom-Kronlöf, Sonn 2007). The need for voice management of informing service have $56 \%$ of users, while $12 \%$ of them have the desire for manage it using the keyboard, $32 \%$ of users want both options. The desire for using Smartphone devices and the related user informing service has $98 \%$ of respondents.

\subsection{Research of the needs of visually impaired persons}

The research was carried out with visually impaired persons who move every day along the traffic network. The planned sample included 175 users, and the survey was completed by 144 users, which is $82 \%$ of the planned number. The research was carried out in the form of online survey and interview, which was done with the users themselves. The importance of providing accurate information to the user about the environment was assessed by $96 \%$ of users as extremely important.

Assessments of the current situation of the informing system at traffic intersections are shown in Figure 4, which means that the users evaluated the current situation as poor or extremely poor.

There are 143 users using mobile devices, which is $99.99 \%$ of the users, and the most frequently used smartphone devices are those of the companies Samsung, Apple, Nokia and HTC devices that are equipped with screen

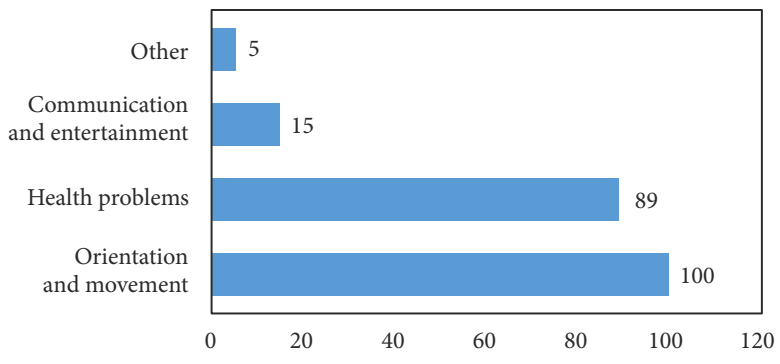

Figure 2. Purpose of using the new service

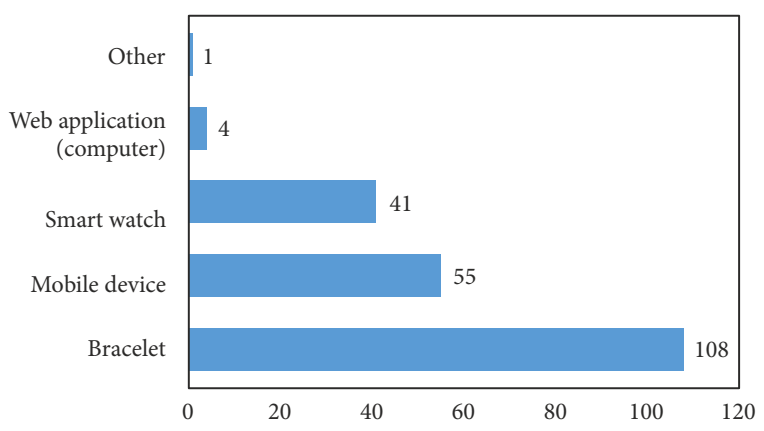

Figure 3. User equipment in the function of informing the users

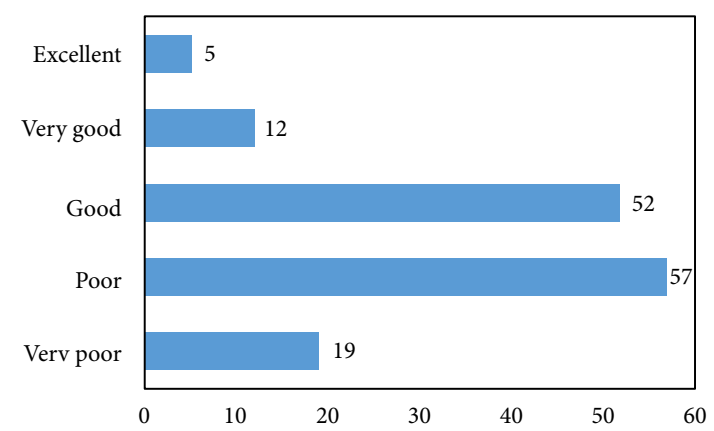

Figure 4. Assessment of the current information system at traffic intersections

readers and content accessibility system for the visually impaired persons.

The mentioned research also included the measuring of the users' movement speed through the sections of the traffic network. The measured users' speed of movement was $0.55 \mathrm{~m} / \mathrm{s}$. Depending on the given movement speed is the user's perception of their safety level in their environment. According to the collected data, only $11 \%$ of users use the navigation and routing services, the reason for such a low number is unsuitable user equipment (device and application solutions) for the visually impaired.

The semaphores system is connected to the user informing system. Light colour information on the pedestrian crossing is realized through the audio signal, with such a way of informing $55 \%$ of the user is dissatisfied. The given time interval for the transition to certain sections of the traffic intersection is also often to short (69\% of users are not satisfied with the current system operation), which further compromised the user's movement safety. Such a form of user informing at the semaphore 
intersection for the purpose of independent movement is important for $98 \%$ of users.

These data represent the basis for defining the user knowledge bases and services, which provide accurate and real-time information to the end user.

\section{Analysis of wireless technologies in order to define user requirements}

User requirements represent the starting point in defining the relevant parameters of the service providing accurate and real-time information to the end user. According to the carried-out research about the users' needs while moving along the traffic network the user requirements should have the following characteristics:

- the simplicity of using hardware components;

- the simplicity of using the applicative solution;

- customisation of the information to the end user;

- the simplicity of the structure of the requested information;

- provision of accurate information and

- education about new services.

In order to define the user requirements, the currently available wireless technologies have been analysed, represented in the AAL concept. Studying the possibilities of the beacon, NFC and RFID technologies has been carried out at the Laboratory of Development and Research of Information and Communication Assistive Technology (Faculty of Transport and Traffic Sciences, University of Zagreb, Croatia). The purpose of using the mentioned technologies is to improve accuracy of determining the user location due to errors generated using GPS technology.

According to Figure 5, the user moves to the POI with the presented direction and scope of signal emission to the user device through which the user receives all relevant information about the environment.

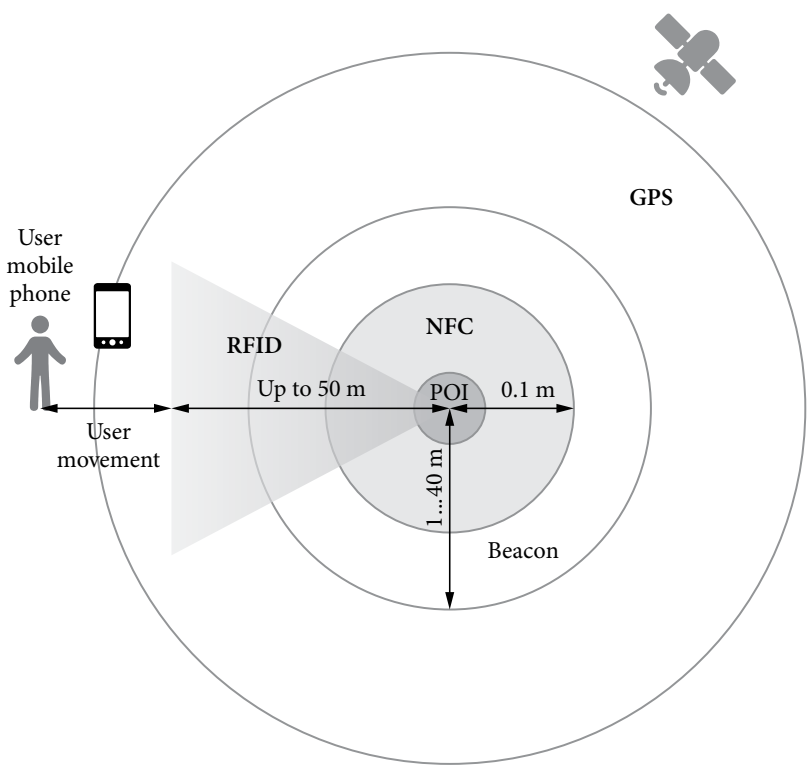

Figure 5. Direction and scope of emitting signals of analysed technologies
Using the GPS technology, it is possible to input the information into POI about the environment, which the user can receive by through their smartphone and application. By using other technologies (NFC or RFID tag and beacon), it is possible to equip additionally the POI by tags that according to the characteristics of the technology can also provide the user with the requested information. According to the characteristics presented in Table 1, the structure of information towards the end user can be defined. The characteristics are directed regarding the size of data storage, connection technology, area of coverage, possibility of work dependent on mobile operating system, working temperature of the tag (optimal), assessment of the data security level and the possibility to operate in IoT environment.

The assessment of the data security level is based on the vulnerabilities and threats related to certain communication technology and their possible advantages. The evaluation of the data security level is in the range from 1 to 5 , where 1 denotes the lowest security level (completely insecure), and 5 the highest security level (completely secure). The tags based on Bluetooth BLE v4.0 communication technology have been evaluated with grade 3 (Panse, T., Panse, P. 2013). The primary reason is the absence of cryptographic methods (due to energy efficiency), which enables the realisation of a large number of threats related with disruption of confidentiality and integrity of data. A large area of signal coverage also provides an easier possibility of unauthorized management of tags from greater distances. The advantage of the mentioned tags is the hardware base, which provides the possibility of implementing cryptographic algorithms that would substantially reduce the number of vulnerabilities.

Tags that use NFC communication technology have been graded with 4 . The vulnerabilities of this type of tags is difficult to use regarding the small area of coverage, which requires manipulation from direct vicinity (Eun et al. 2013). The hardware simplicity of design can be observed also as a vulnerability (lack of possibility of implementing robust security methods), but also as an advantage since a simpler design means fewer vulnerabilities. Grade 2 was assigned to tags based on RFID communication technology. The grade of the data security level in this type of tags is based on a large number of vulnerabilities and assumed threats (Farooq et al. 2015).

The tags that are subject to standard NFC Forum Type 3 and NFC Forum Type 4 support the symmetric cryptographic algorithms: Advanced Encryption Standard (AES) and Data Encryption Standard (DES); however, tags analysed by this paper support the standard NFC Forum Type 2 as well as a large number of tags available on the market. The mentioned types of tags have been analysed from the aspect of the possibility of information storage in outdoor environment in which tests have been carried out - according to their characteristic dimensions the indicated tags are suitable for the implementation on micro locations such as the pedestrian crossing (Panse, T., Panse, P. 2013). In case of the mentioned tags the resilience to me- 
Table 1. Analysis of the characteristics of tags depending on the connecting technology

\begin{tabular}{|l|l|c|l|l|c|c|}
\hline \multicolumn{1}{|c|}{ Type of tags } & \multicolumn{1}{|c|}{$\begin{array}{c}\text { Connecting } \\
\text { technology }\end{array}$} & $\begin{array}{c}\text { Data storage } \\
\text { capacity [KB] }\end{array}$ & $\begin{array}{c}\text { Area of } \\
\text { coverage [m] }\end{array}$ & $\begin{array}{c}\text { Mobile operating } \\
\text { system }\end{array}$ & $\begin{array}{c}\text { Data } \\
\text { security } \\
\text { level }\end{array}$ & $\begin{array}{c}\text { Possibility of operation } \\
\text { in IoT environment }\end{array}$ \\
\hline Beacon Kontakt.io & Bluetooth BLE v4.0 & 256 & $2 \ldots 70$ & Android; iOS & 3 & Yes \\
\hline Beacon SmartBeacon & Bluetooth BLE v4.0 & 256 & $2 \ldots 50$ & Android; iOS & 3 & Yes \\
\hline NTAG 203 & NFC & 0.168 & Up to 0.10 & Android; WP 8.1 & 4 & Yes \\
\hline MIFARE Ultralight & NFC & 0.064 & Up to 0.10 & Android; WP 8.1 & 4 & Yes \\
\hline NTAG213 All surface & NFC & 0.180 & Up to 0.05 & Android; WP 8.1 & 4 & Yes \\
\hline FeliCa Lite & NFC/RFID & 0.224 & Up to 0.10 & Android; WP 8.1 & 4 & Yes \\
\hline I-Code 2 label & NFC/RFID & 0.112 & Up to 1.5 & Android; WP 8.1 & 4 & Yes \\
\hline RoHS Active & RFID & - & $40 \ldots 80$ & - & 2 & Yes \\
\hline
\end{tabular}

Table 2. Analysis of the characteristics of tags depending on the connecting technology

\begin{tabular}{|c|c|c|}
\hline Type of tags & Possibility of operation in weather conditions & Charging lifecycle \\
\hline Beacon Kontakt.io & $\begin{array}{l}\text { All weather conditions and possibility of } \\
\text { operation under water up to } 10 \mathrm{~m}\end{array}$ & $\begin{array}{l}\text { Depending on the defined operating distance: } \\
-4 \mathrm{~m}-2 \text { years; } \\
-35 \mathrm{~m}-\text { up to } 2 \text { years and } \\
-70 \mathrm{~m}-\text { up to } 15 \text { months }\end{array}$ \\
\hline Beacon SmartBeacon & Dry weather conditions & 2 years \\
\hline NTAG 203 & All weather conditions & Passive tag (no battery) \\
\hline MIFARE Ultralight & Minimal resilience - dry weather & Passive tag (no battery) \\
\hline NTAG213 All surface & All weather conditions & Passive tag (no battery) \\
\hline FeliCa Lite & All weather conditions & Passive tag (no battery) \\
\hline I-Code 2 label & Minimal resilience - dry weather & Passive tag (no battery) \\
\hline
\end{tabular}

teorological conditions (sun, rain, snow, ice) is important, as well as the operating lifecycle of charging as shown in Table 2 .

According to the carried out analysis the user in the vicinity of POI of pedestrian crossing and destination arrival, and upon entering the vehicle (tram or bus) sometimes experiences difficulties in the location of NFC tag. This problem occurs due to the vicinity that is necessary in order to provide the user with the requested information. When using RFID technology, apart from their mobile terminal device the user must also have an active tag (RoHS Compliant 433MHz Active RFID Tag), which serves for identification in the traffic environment. Therefore, with the purpose of designing the user information service, it is suggested to use the beacon Bluetooth Low Energy (BLE) technology and to apply the model of assistive technologies systems (Manduchi, Kurniawan 2013).

\section{Traffic network model equipped with beacon technology}

The system architecture that allows users informing is based on the CC concept. The user's environment is equipped with beacon devices whereas the user equipment is the smartphone device and specialized application (Android OS), which provides the information service.

The traffic intersection is equipped with beacon devices as presented in Figure 6. In the beacon devices configuration, the traffic intersections have their major values $1,2,3$, which defines the class of the beacon device depending on the coverage location.

The user moves along a traffic intersection equipped with beacon devices (B1...B58), which are identified by the user's smartphone according to previously defined requirements in the mobile application and the user receives information about the environment.

The network of beacon devices that were used to perform the research are presented in Figure 7. Beacon devices B24 and B25 are set on a bus and a tram stop on the user's movement route. The methods of movement along the traffic network are defined by the user through applicative solution on their smartphone or web portal, where the applicative solution uses the beacon network to provide information to the user. Until the location of identifying the beacon device B1 the user uses GPS technology. Beacon device recognizes the user who uses the mobile terminal device and the applicative solution at a distance of $70 \mathrm{~m}$ with possible error of $\sim 10 \mathrm{~cm}$, which was measured while analysing the beacon technology.

In order to simulate the model of user moving along the traffic network, a simulation model in MATLAB tools has been developed. A model of the traffic network has been created in it, along which users move every day regardless of the transport mode. The change of traffic mode is presented in Figure $7 \mathrm{~b}$, where the user can move as a passenger or use bus and tram transport (their stops are also equipped with beacon devices). In the respective model, the nodes are represented by beacon devices, set 


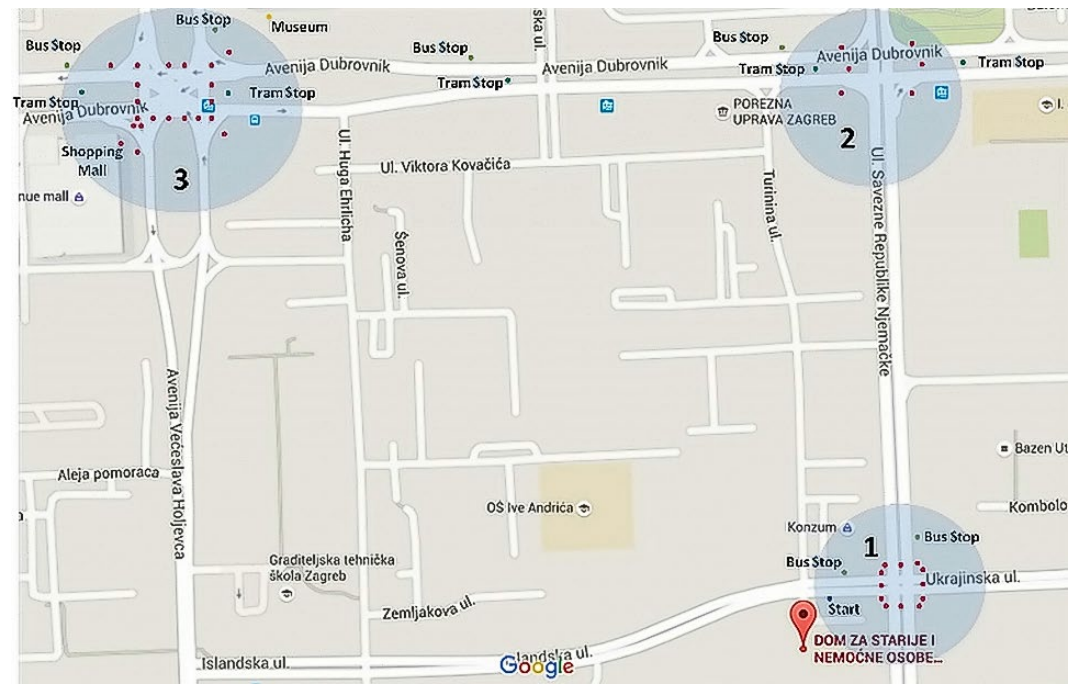

Figure 6. Movement of user in traffic environment

as shown in Figures 7. Figure 8 shows the implementation of MATLAB in the process of simulating the determination of the shortest route for the user, from point: home for the elderly - B1 to the point of the "Museum of Contemporary Arts" B26.

The architecture of the users informing system by means of a beacon device is based on the CC for the blind concept (Periša et al. 2014), where the beacon device apart from major uses also the Universally Unique IDentifiers (UUID) value providing the user with the information about the environment. Major and UUID values represent a very important component in Beacon tagging. Major values are used for identification of a group, for example, all beacons on a particular traffic intersection could be assigned a unique major value. The UUID is identifying system, which allows a unique number to be generated for a device.

Figure 9 shows the architecture of the information system and an example of the record based on which the Internet Service Provider (ISP) via mobile application generates to the user relevant real-time information about their environment, based on beacon location.

The mentioned architecture makes it possible for the user to create the route of movement by using a customised web portal, at the same time being able to provide the user with other information as well, e.g. the nearby stores, public institutions, recreational facilities, etc. The beacon technology provides the user with dynamic content of information or real-time informing, which means informing of the user if there is an unexpected event occurring on their defined route, e.g. works on the pedestrian path, which can be an extremely important information for a visually impaired person. The information system as presented by the system architecture includes the participation of other stakeholders as well such as the public urban transport system, Croatian Automobile Club, automatic control system and other interest groups (societies of disabled persons and elderly persons). a)

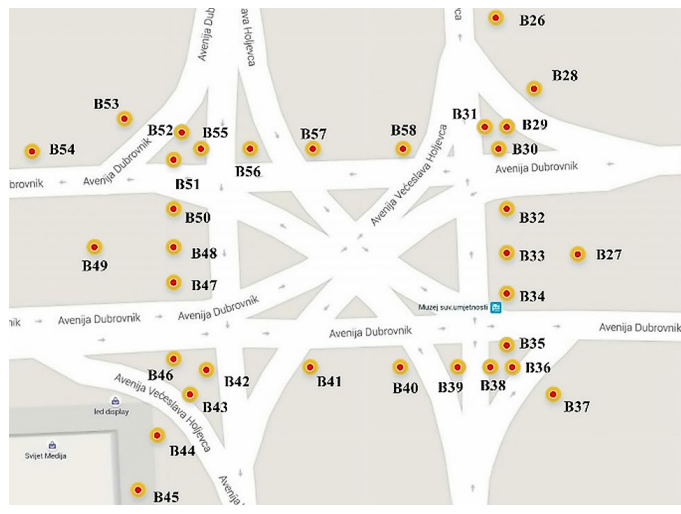

b)

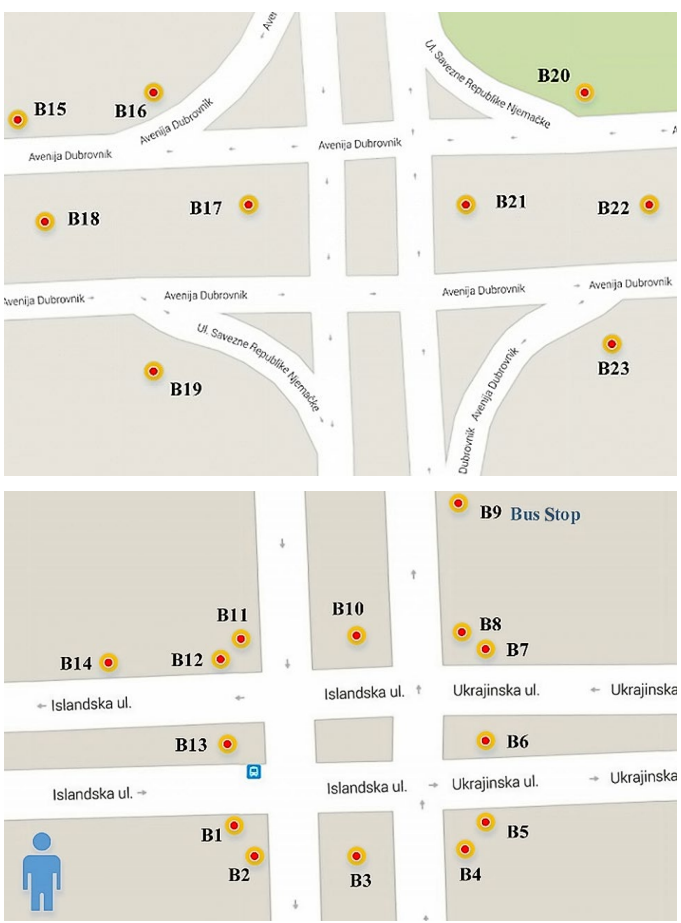

Figure 7. Network of beacon devices: a - starting point of movement; $b$ - possibility of transport mode change; $c$ - complex traffic intersection in the vicinity of various areas of interest 


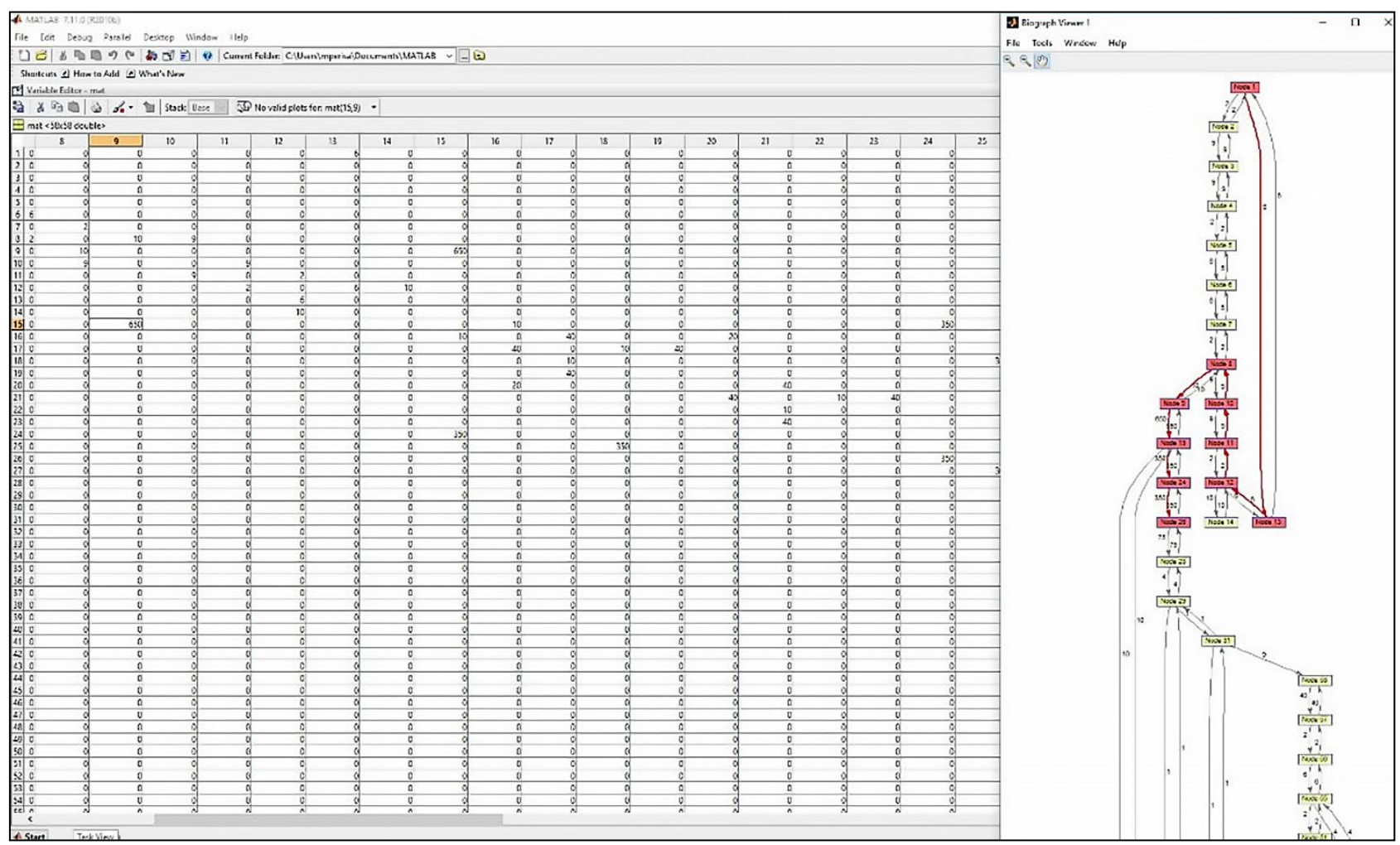

Figure 8. Application of MATLAB for the simulation of a traffic network based on beacon nodes tagging

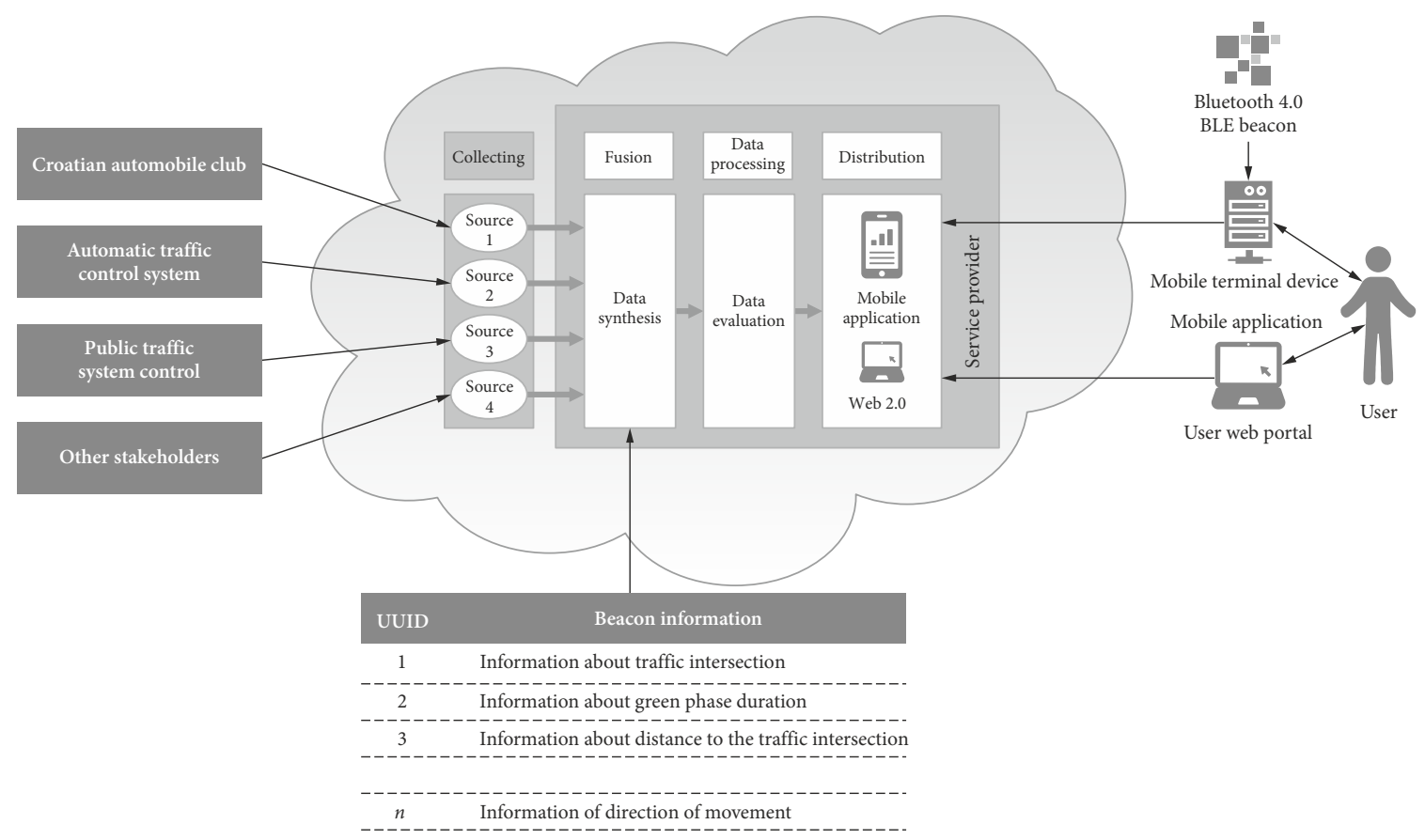

Figure 9. Architecture of user information system

In CC environment the data of all stakeholders are collected, followed by an important process of customisation and fusion of data to the defined work environment (type of database, output values). The process of data evaluation assigns the value of importance of the message that needs to be delivered to the end user. An example is the informing of the user about a new event that occurred on the defined movement route if there are infrastructural works on it. The data can be distributed via web environment or mobile terminal device in real environment, which includes also the beacon devices.

According to the collected information on user experience of using information and communication technology, it is important to design a service according to content 


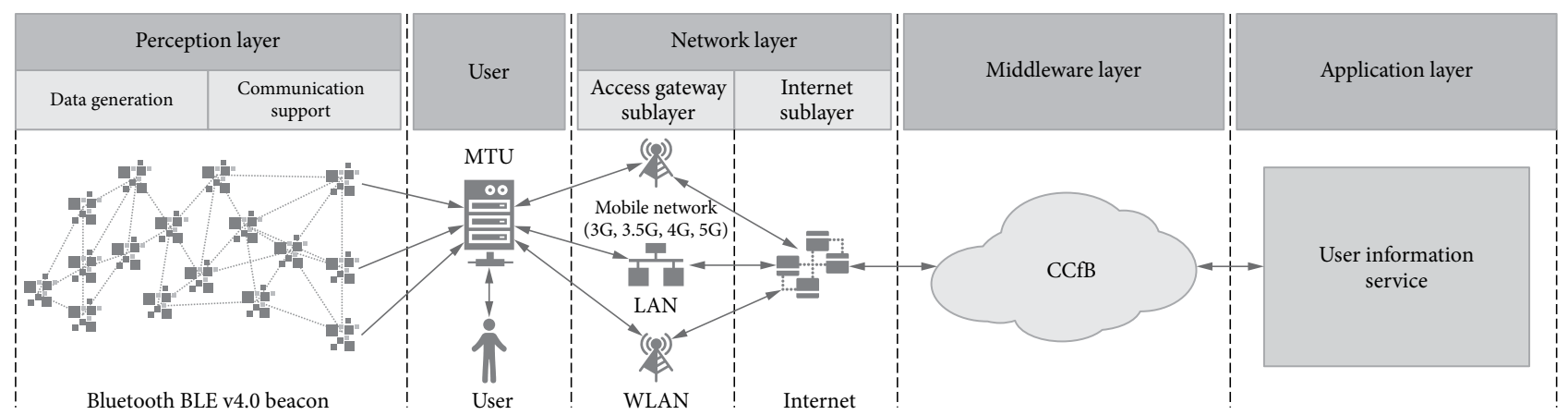

Figure 10. Classification of the security risk of the layers of the proposed architecture

accessibility elements. For this purpose, it is important to meet the following customer requirements for delivering real-time customer service:

- content customization regardless of the user damage degree;

- accurate information delivery;

- system customization according to the user's speed of movement;

- development for the most used mobile operating systems and

- possibility of speech management in the user's native language.

According to the proposed system architecture and user requirements, data security and protection is also important, and the security risk classification is explained by using this architecture in the IoT environment.

\section{Classification of the security risk of the proposed IoT architecture}

In the presented system architecture, the IoT concept has been implemented, realised by means of layered architecture consisting of perception, network, middleware, and application layer. The perception layer consists of two basic functionalities, generation of data and collaboration among elements of the respective layer (Jing et al. 2014). In the system of informing the users proposed by this paper, the perception layer (Bluetooth beacon) is in direct communication with the user's mobile terminal device.

The received data are transferred by the mobile terminal device via communication infrastructure to the middleware layer. The middleware layer has the task to process the collected data and to transfer them then to the application layer, which then presents them to the user in a user-understandable form.

The security risk of a single layer is assigned from level low (lowest risk of disruption of the security principles) to high (maximum risk of disruption of security principles) presented by Figure 10. The perception layer is classified by the highest level of security risk high, i.e. maximum level of risk due to the specific characteristic of the observed layer and the vulnerability such as big hardware restrictions, physical dimensions, and lack of physical protection of tags. The access layer of the network layer is classified by the level of security risk low-to-medium due to the known drawbacks such as wireless data transfer and a huge number of threats directed to this sublayer. The advantages of this sublayer are continuous studies of the protection methods as well as their implementation. The Internet sublayer is classified with the minimum level of security risk low due to extremely complex extraction of data that use it as a medium to be transferred to the middleware layer. Medium level of security risk classifies the middleware layer on which Cloud Computing for the Blind (CCfB) is based.

The reasons lie in a large number of users and data that are processed and stored in the observed layer as well as the known vulnerabilities of virtualisation whose unauthorized usage has the potential to cause damage of great dimensions to a large number of users at a time. The classification of risk to the user information system in the traffic environment is based on the increased implementation of the IoT concept in certain environment according to which the level of risk Medium has been assigned (Cvitić et al. 2016).

\section{Results and discussion}

The aim of this research is to propose a system architecture for informing the users who move along the traffic network using Bluetooth BLE v4.0 technology, beacon devices and CC concept. According to the carried out analysis of the technologies that currently have the possibility of providing information of the beacon technologies due to their technical specifications has proven as the best choice in order to provide reliable and accurate information.

To test the acceptance of the proposed service by the users, a survey has been performed among the users, where possible functionalities of the information service have been described to the users. The results indicate that $68 \%$ of users responded that they would use the mentioned service. It is worth mentioning that for this question also the users who do not use mobile terminal devices have been included in the survey and because of the described service the number of the interested users increased.

The interest points enabled by GPS technology by being input into the map and the movement route due to error in locating may additionally threaten the security 
of user movement. Using beacon technology, the risk of such error is much smaller, which has been proven by this research. In case of using NFC technology in order to provide information in outdoor spaces there is the problem of non-standardization of keys, which can be equipped with the mentioned technology.

The standardization refers to the place of implementation of the device that the user should locate at a distance of $10 \mathrm{~cm}$ in order to obtain all the requested information. In order to use RFID technology, apart from mobile terminal device, the user should also use an active tag for identification, which for a large number of users means a complication in usage. The defined architecture based on beacon technology in this research is based on IoT concept thus enabling data accessibility at any moment regardless of the location 24/7.

\section{Conclusions}

The proposed system architecture and its functionalities offer the user various classes of real-time information, thus achieving the accuracy and timeliness of user information. The contribution of this research is reflected in the increase of the user movement safety, satisfaction of the citizens and improvement in the quality of living.

Apart from contributing to better usage of the currently available technology in indoor spaces, the AAL concept can do this also in outdoor spaces. The beacon technology in this case represents the basis for the identification of the mobile object and with synergic action with the applicative solution and cloud architecture it enables real-time informing of the users. This technology has the possibility of operating with all mobile operating systems (Android, iOS, Windows Phone), which is also an advantage compared to the pervious solutions. The Bluetooth BLE v4.0 technology, compared to the previous versions of Bluetooth technology and GPS technology, features a much lower battery consumption thus making this service more reliable.

The Internet portal based on Web 2.0 technology enables customization of the requested information according to the level of user's impairment. The mentioned research represents the basis for the concept of new services based on the AAL concept, universal design principles and models of assistive technology systems.

\section{References}

Boualouache, A. E.; Nouali, O.; Moussaoui, S.; Derder, A. 2015. A BLE-based data collection system for IoT, in 2015 First International Conference on New Technologies of Information and Communication (NTIC), 8-9 November 2015, Mila, Algeria, 1-5. https://doi.org/10.1109/NTIC.2015.7368748

Brilhault, A.; Kammoun, S.; Gutierrez, O.; Truillet, P.; Jouffrais, C. 2011. Fusion of artificial vision and GPS to improve blind pedestrian positioning, in 2011 4th IFIP International Conference on New Technologies, Mobility and Security, 7-10 February 2011, Paris, France, 1-5.

https://doi.org/10.1109/NTMS.2011.5721061
Brinkmann, M.; Floeck, M.; Litz, L. 2008. Concept and design of an AAL home monitoring system based on a personal computerized assistive unit, Communications in Computer and Information Science 11: 218-227.

https://doi.org/10.1007/978-3-540-85379-4_27

Cheng, R.-S.; Hong, W.-J.; Wang, J.-S.; Lin, K. W. 2016. Seamless guidance system combining GPS, BLE beacon, and NFC technologies, Mobile Information Systems 2016: 5032365. https://doi.org/10.1155/2016/5032365

Cvitić, I.; Vujić, M.; Husnjak, S. 2016. Classification of security risks in the IoT environment, in Proceedings of the 26th DAAAM International Symposium on Intelligent Manufacturing and Automation, 21-24 October 2015, Zadar, Croatia, 0731-0740.

https://doi.org/10.2507/26th.daaam.proceedings.102

Dhananjeyan, S.; Sundaram, K. M.; Kalaiyarasi, A.; Kuppusamy, P. G. 2016. Design and development of blind navigation system using GSM and RFID technology, Indian Journal of Science and Technology 9(2): 1-5. https://doi.org/10.17485/ijst/2016/v9i2/85809

Eun, H.; Lee, H.; Oh, H. 2013. Conditional privacy preserving security protocol for NFC applications, IEEE Transactions on Consumer Electronics 59(1): 153-160.

https://doi.org/10.1109/TCE.2013.6490254

Farooq, M. U.; Waseem, M.; Khairi, A.; Mazhar, S. 2015. A critical analysis on the security concerns of Internet of Things (IoT), International Journal of Computer Applications 111(7): 1-6. https://doi.org/10.5120/19547-1280

Garcia, N. M.; Rodrigues, J. J. P. C. 2015. Ambient Assisted Living. CRC Press.

Häggblom-Kronlöf, G.; Sonn, U. 2007. Use of assistive devices a reality full of contradictions in elderly persons' everyday life, Disability and Rehabilitation: Assistive Technology 2(6): 335-345. https://doi.org/10.1080/17483100701701672

Jing, Q.; Vasilakos, A. V.; Wan, J.; Lu, J.; Qiu, D. 2014. Security of the Internet of Things: perspectives and challenges, Wireless Networks 20(8): 2481-2501. https://doi.org/10.1007/s11276-014-0761-7

Joseph, S. L.; Xiao, J.; Zhang, X.; Chawda, B.; Narang, K.; Rajput, N.; Mehta, S.; Subramaniam, S. V. 2015. Being aware of the world: toward using social media to support the blind with navigation, IEEE Transactions on Human-Machine Systems 45(3): 399-405. https://doi.org/10.1109/THMS.2014.2382582

Korial, A. E.; Abdullah, M. N. 2016. Novel method using beacon and smart phone for visually impaired/blind people, International Journal of Computer Applications 137(1): 33-39. https://doi.org/10.5120/ijca2016908674

Loomis, J. M.; Golledge, R. G.; Klatzky, R. L. 2016. Navigation system for the blind: auditory display modes and guidance, Presence: Teleoperators and Virtual Environments 7(2): 193204. https://doi.org/10.1162/105474698565677

Manduchi, R.; Kurniawan, S. 2013. Assistive Technology for Blindness and Low Vision. CRC Press. 441 p.

Panse, T.; Panse, P. 2013. A survey on security threats and vulnerability attacks on Bluetooth communication, International Journal of Computer Science and Information Technologies 4(5): 741-746.

Peraković, D.; Periša, M.; Bukljaš Skočibušić, M. 2015a. Possibilities of implementing ambient assisted living concept in traffic environment, Archives of Transport System Telematics 8(1): 30-34.

Peraković, D.; Periša, M.; Remenar, V. 2015b. Model of guidance for visually impaired persons in the traffic network, Transportation Research Part F: Traffic Psychology and Behaviour 31: 1-11. https://doi.org/10.1016/j.trf.2015.03.007 
Periša, M. 2013. Dinamičko vođenje i usmjeravanje slijepih i slabovidnih osoba u prometu [Dynamic Guiding and Routing of Disabled and Visually Impaired Persons in Traffic]: Doctoral Thesis. University of Zagreb, Croatia. 205 s. (in Croatian).

Periša, M.; Peraković, D.; Šarić, S. 2014. Conceptual model of providing traffic navigation services to visually impaired persons, Promet - Traffic \& Transportation 26(3): 209-218. https://doi.org/10.7307/ptt.v26i3.1492

Periša, M.; Peraković, D.; Vaculík, J. 2015. Adaptive technologies for the blind and visual impaired persons in the traffic network, Transport 30(3): 247-252.

https://doi.org/10.3846/16484142.2014.1003405

Ramirez, A. R. G.; González-Carrasco, I.; Jasper, G. H.; Lopez, A. L.; Lopez-Cuadrado, J. L.; García-Crespo, A. 2017. Towards human smart cities: internet of things for sensory impaired individuals, Computing 99(1): 107-126. https://doi.org/10.1007/s00607-016-0529-2

Spinsante, S.; Gambi, E. 2015. NFC-based user interface for smart environments, Advances in Human-Computer Interaction 2015: 854671. https://doi.org/10.1155/2015/854671

Van der Bie, J.; Visser, B.; Matsari, J.; Singh, M.; Van Hasselt, T.; Koopman, J.; Kröse, B. 2016. Guiding the visually impaired through the environment with beacons, in UbiComp'16: Proceedings of the 2016 ACM International Joint Conference on Pervasive and Ubiquitous Computing: Adjunct, 12-16 September 2016, Heidelberg, Germany, 385-388.

https://doi.org/10.1145/2968219.2971387 